\title{
Shaping Perovskites: In Situ Crystallization Mechanism of Rapid Thermally Annealed, Prepatterned Perovskite Films
}

\author{
Antonio Günzler, Esteban Bermúdez-Ureña, Loreta A. Muscarella, Mario Ochoa, Efraín Ochoa-Martínez, \\ Bruno Ehrler, Michael Saliba,* and Ullrich Steiner*
}

Cite This: ACS Appl. Mater. Interfaces 2021, 13, 6854-6863

Read Online

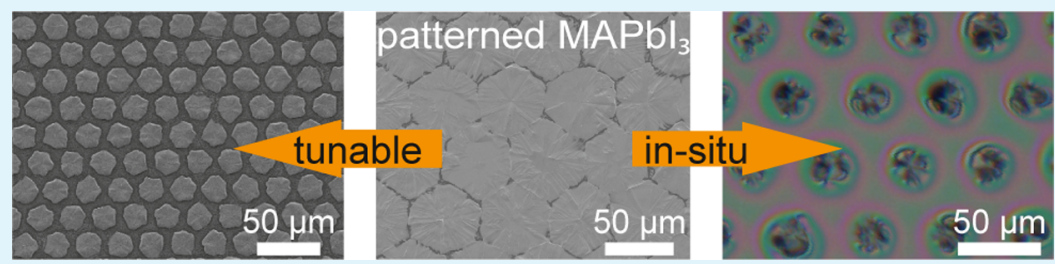

ABSTRACT: Understanding and controlling the crystallization of organic-inorganic perovskite materials is important for their function in optoelectronic applications. This control is particularly delicate in scalable single-step thermal annealing methods. In this work, the crystallization mechanisms of flash infrared-annealed perovskite films, grown on substrates with lithographically patterned $\mathrm{Au}$ nucleation seeds, are investigated. The patterning enables the in situ observation to study the crystallization kinetics and the precise control of the perovskite nucleation and domain growth, while retaining the characteristic polycrystalline micromorphology with larger crystallites at the boundaries of the crystal domains, as shown by electron backscattering diffraction. Time-resolved photoluminescence measurements reveal longer charge carrier lifetimes in regions with large crystallites on the domain boundaries, relative to the domain interior. By increasing the nucleation site density, the proportion of larger crystallites is increased. This study shows that the combination of rapid thermal annealing with nucleation control is a promising approach to improve perovskite crystallinity and thereby ultimately the performance of optoelectronic devices.

KEYWORDS: perovskite patterning, in situ, flash infrared annealing, crystallization, nucleation

\section{INTRODUCTION}

Perovskite solar cells (PSCs) have seen a rapid increase in power conversion efficiency (PCE) over the past decade of research to $25.5 \%$ now, approaching that of established technologies. $^{1-4}$ Reaching ever closer to the theoretical Shockley-Queisser limit at PCEs of $\sim 33 \%$, $^{5,6}$ increasing focus has shifted toward the long-term stability (i.e., device lifetime) of PSCs and possible strategies for upscaling from a lab- to industry-scale production. ${ }^{7}$ The commonly employed antisolvent method, where an antisolvent is dripped onto the liquid precursor, however, is challenging to scale-up at a potentially high economic and ecological cost. ${ }^{8}$

Drawing inspiration from the market-leading silicon-based photovoltaics (PVs), large single-crystal active layers are a proposed ideal. ${ }^{9}$ However, challenges remain related to the incorporation of perovskite single crystals into the overall device architecture and ensuring optimal contacts to the electron/hole selective layers to reach polycrystalline PCEs, ${ }^{10}$ and the fabrication of single-crystal perovskite devices is often still complex. ${ }^{11,12}$ Whereas high-end fabrication methods, for instance, metal-organic chemical vapor deposition or hybrid vapor phase epitaxy, bring other crystalline thin-film PV technologies close to PCEs of $30 \%,{ }^{1,13,14}$ new strategies are required to push for such efficiencies in PSCs.
Therefore, in order to maintain the advantages of solutionbased processing and controlling the film crystallinity, a major challenge is to fundamentally understand and control the crystallization processes when the perovskite active layer is formed from its precursors, most commonly from a solution or vapor. $^{7,15-17}$ Although perovskites show a remarkable tolerance to defects, ${ }^{18}$ it has also been observed that the boundaries of individual crystallites (often referred to as grains) are frequently the origin of degradation processes. ${ }^{19}$ Strategies to mitigate performance losses include precursor additives to control morphology, passivation with polymeric nanolayers, and stoichiometric tuning of the perovskite, all of which come with increased complexity and drawbacks. ${ }^{20-24}$ A comprehensive understanding of perovskite film growth mechanisms is not only critical for the benefit of solar cell applications but also for other emerging perovskite-based light technologies such as light-emitting devices, detectors, lasers, and sensors, for

Received: November 24, 2020

Accepted: January 13, 2021

Published: January 29, 2021 

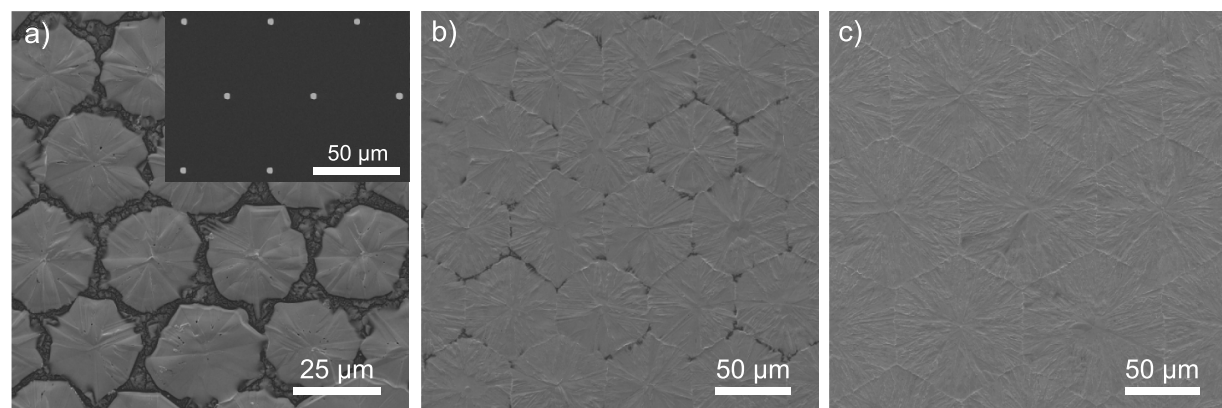

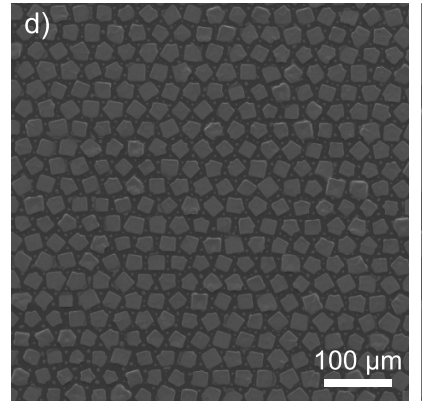

g)

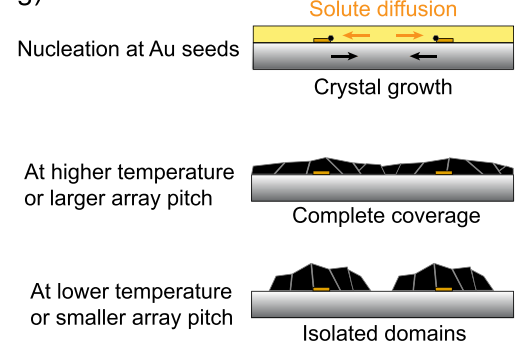

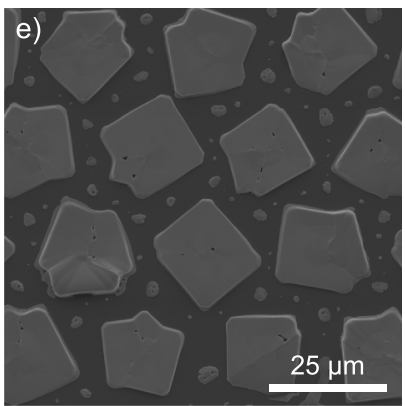

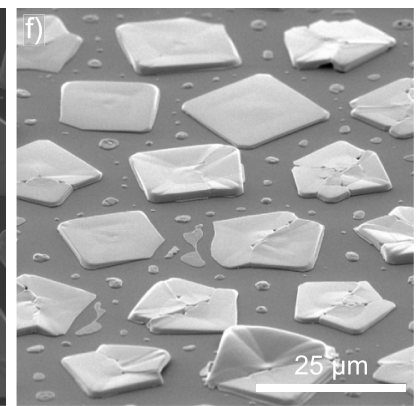

h)

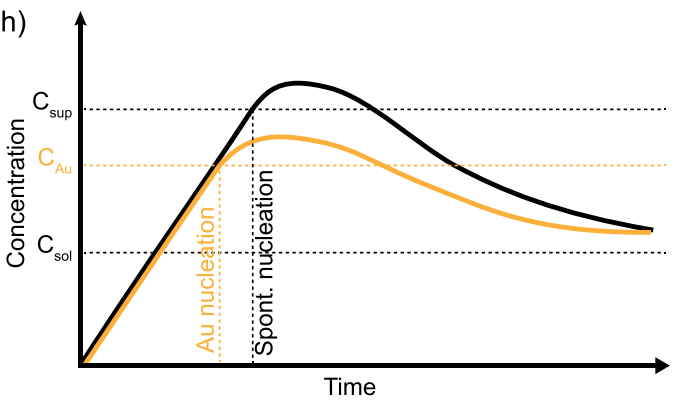

Figure 1. $\mathrm{MAPbI}_{3}$ samples annealed by FIRA on Au seed-patterned Si substrates. Au seeds function as predetermined nucleation sites patterned as a hexagonal lattice with varying pitches of (a) $30 \mu \mathrm{m}$ (inset: Au-patterned Si substrate), (b) 60, and (c) $90 \mu \mathrm{m}$. The inset in (a) shows the patterned substrate. (d-f) SEM images of FIRA-MAPbBr 3 on Au-patterned Si substrate with a $25 \mu \mathrm{m}$ pitch: (d) top view overview, (e) close-up, and (f) tilted at $45^{\circ}$. (g) Schematic illustration of nucleation, crystal growth, and solute diffusion for different parameters. (h) LaMer curve for the spontaneous and $\mathrm{Au}$-seeded nucleation, indicating the lowered concentration threshold for the latter.

many of which patterning of the perovskite material has gained considerable attention. ${ }^{25-27}$

In this work, we employ a model system that spatially predetermines nucleation sites for perovskite films annealed by the flash infrared annealing (FIRA) method. ${ }^{28,29}$ This approach is also suitable for other antisolvent-free thermal annealing methods compatible with large-scale device production (e.g., meniscus-assisted printing), ${ }^{30}$ where the crystallization process is exclusively driven by thermal evaporation of the solvents. ${ }^{31,32}$ Methylammonium lead triiodide $\left(\mathrm{MAPbI}_{3}\right)$, one of the most studied perovskite compositions, was selected here to demonstrate the facile crystallization control. The FIRA method, yielding highly efficient $\mathrm{MAPbI}_{3}$ PSCs, was used to anneal $\mathrm{MAPbI}_{3}$ films on substrates with photolithographically patterned arrays of $\mathrm{Au}$ nucleation seeds. ${ }^{33}$ The resulting morphologies are then characterized using electron backscattering diffraction (EBSD) and time-resolved photoluminescence (TRPL) mapping. Other perovskite compositions were then investigated in the same manner to further elucidate the crystallization dynamics.

To gain further insight into the morphology evolution of perovskite films, we combine the deterministic nucleation-site patterning with an in situ observation method by optical microscopy. ${ }^{34}$ The patterned nucleation seeds enable us to identify the location of nucleation a priori in order to investigate the nucleation and crystal growth dynamics of the perovskite films. We also show that the method of prepatterned nucleation sites can be generalized to other commonly used perovskite compositions.

\section{RESULTS AND DISCUSSION}

2.1. Prepatterned Perovskite Films Annealed by FIRA. FIRA is a rapid antisolvent-free annealing method that produces perovskite films with distinct polycrystalline morphologies that exhibit large polycrystalline domains. Each of these domains forms from a nucleation site, and they produce a weighted Voronoi tessellation according to the geometry of the nucleation sites. ${ }^{28,35}$ The weighting in the Voronoi tessellation results from a temporal variance of the spontaneous supersaturation-driven nucleation, where spontaneous nucleation can be both homogeneous nucleation from the precursor solution and heterogeneous nucleation caused by contaminants, the substrate surface, or the liquid-gas interface. $^{36}$ Each domain has a characteristic intrinsic morphology consisting of small individual crystallites in the center close to the nucleation site and larger crystallites close to the boundaries of the domains. ${ }^{37}$ The diameters of the domains are typically in the $100-300 \mu \mathrm{m}$ range. ${ }^{28}$ 


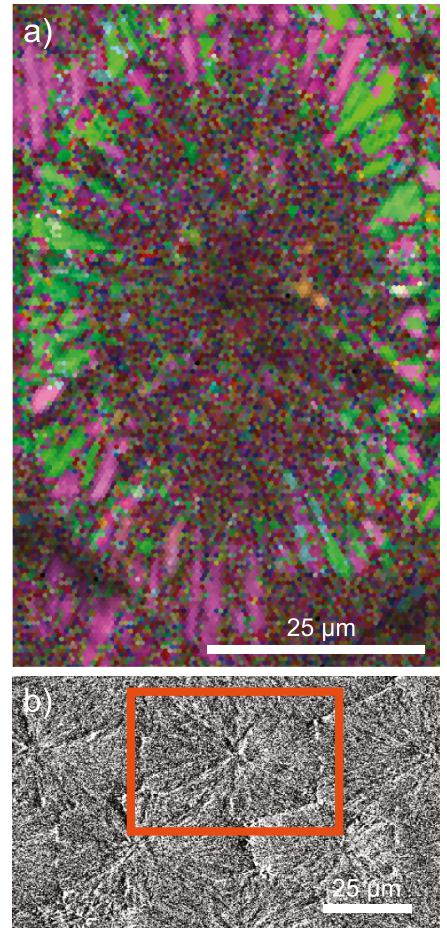

c)

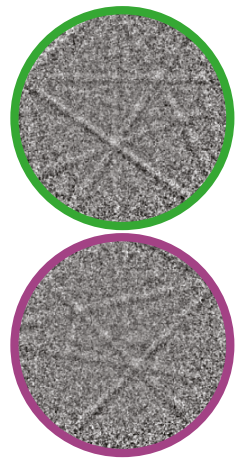

e)

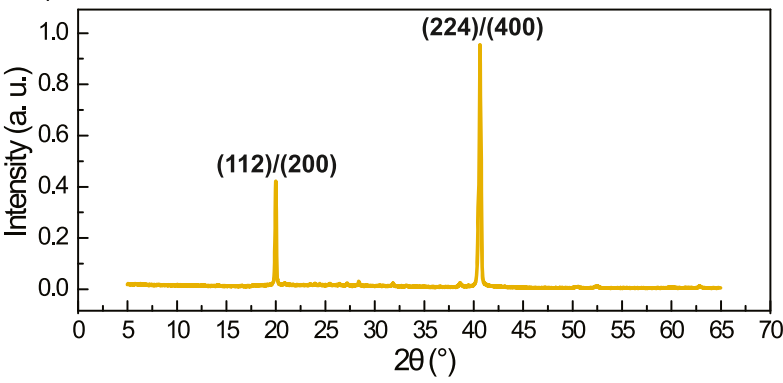

Figure 2. Micromorphology of rapid thermally annealed $\mathrm{MAPbI}_{3}$ crystal domains. (a) EBSD map indicating the crystallographic orientations in $z$ direction. (b) SEM image indicating the measured area within the pattern for EBSD mapping. (c) Kikuchi patterns identifying the two dominant crystallographic orientations. (d) Distribution of crystallographic orientations over the measured area. (e) XRD data for a prepatterned $\mathrm{MAPbI}_{3}$ film on an ITO-coated glass substrate.

The domain nucleation sites can be spatially predetermined by patterning silicon and tin-doped indium oxide (ITO)coated glass substrates with hexagonal arrays of Au seeds, as demonstrated by Geske et al. ${ }^{33}$ They hypothesize a lowering interfacial energy at the Au surface caused by a catalytic effect of $\mathrm{Au}$ to give rise to the nucleation effect. The details of this nucleation effect are, however, currently unclear.

In the present study, the reported patterning method is combined with the FIRA deposition and annealing protocol for the manufacture of prepatterned $\mathrm{MAPbI}_{3}$ and methylammonium lead tribromide $\left(\mathrm{MAPbBr}_{3}\right)$ films, as previously established by Sánchez et al., ${ }^{29}$ that has demonstrated competitive device performances. The prepatterned perovskite films of this study, therefore, resemble the nucleation and crystallization kinetics under deposition parameters relevant to the rapid thermally annealed high-performance PSCs.

Figure $1 \mathrm{a}-\mathrm{c}$ and $\mathrm{d}-\mathrm{f}$ show $\mathrm{MAPbI}_{3}$ and $\mathrm{MAPbBr}_{3}$ films annealed by FIRA, respectively, for various pitch sizes of predetermined nucleation sites (Au patches on Si substrate, as shown in the inset in Figure 1a). By varying the distance between the $\mathrm{Au}$ seeds, the density of the nucleation sites, and thus the shape and size of the domain, can be controlled. Siand ITO-coated glass substrates were prepatterned to obtain compact perovskite films with hexagonal domains of tunable diameters in the $30-100 \mu \mathrm{m}$ range. The geometric regularity of the domains within the patterns shows that predetermined nucleation has very little temporal variance, that is, all nuclei form almost simultaneously, producing an unweighted Voronoi tessellation, whenever sufficient precursor material is available for the domains to grow until they meet. At spacings larger than $100 \mu \mathrm{m}$, spontaneous nucleation was observed, forming perovskite domains between the Au-seeded domains. At patch distances of $30 \mu \mathrm{m}$ and below, the diffusion of precursor molecules toward the crystallisation front leads to depleted areas between the isolated domains, as shown in Figure 1a,d-f. Note, however, that despite the precursor depletion at a seed pitch of $30 \mu \mathrm{m}$, as in Figure 1a, some of the domains appear to have hexagonal shapes. This is due to a surface tension and convection effect in the precursor solution during thermal annealing, which is further discussed below.

The depletion results from the vicinity of the seeded nucleation sites, as a higher density of growing crystal domains compete for precursor molecules. As the annealing conditions with FIRA were kept identical, the different film morphologies solely depend on the pitch of the patterned hexagonal Au seed arrays. Because the nucleation and crystal growth locally remove solutes from the solution, a concentration gradient is created with a lower solute concentration at the crystal growth front than in the bulk solution. Precursor molecules, therefore, diffuse toward the growing domains, which can lead to depleted areas for sufficiently small pitch sizes or low annealing temperatures, where thermal solvent evaporation does not outpace solute diffusion (see schematic in Figure 1g). At higher temperatures, crystal growth parallel to the substrate outpaces solute diffusion because of fast solvent evaporation. At the same time, increasing the array pitch, that is, the distance between $\mathrm{Au}$ seeds, results in a lower density of patterned nucleation sites competing for solutes and thus no complete solute depletion between the domains. For $\mathrm{MAPbBr}_{3}$, the depletion effect is more prominent than for $\mathrm{MAPbI}_{3}$, leading to entirely isolated domains that consist of only a few individual single crystals, even at array pitch distances of $50 \mu \mathrm{m}$, resulting in isolated platelets and partially connected domains (Figure S1). The cubic crystal lattice of $\mathrm{MAPbBr}_{3}$ probably enables the growth of oriented cuboid 
(a)

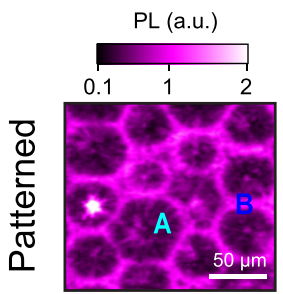

(b)

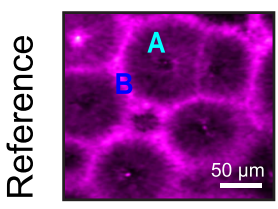

(c)

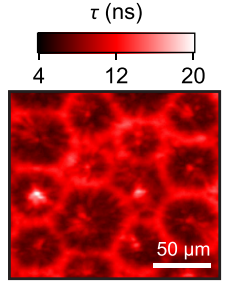

(d)

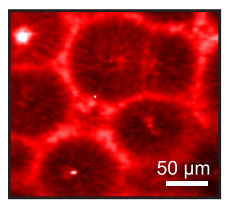

(e)

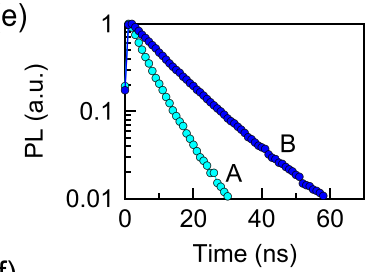

(f)

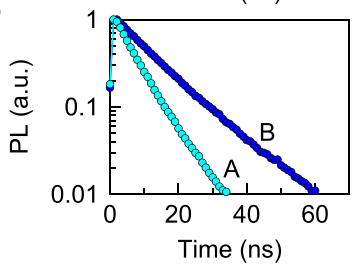

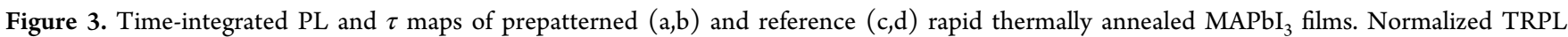
decays at two different locations (A: domain interior and B: near-domain boundary) indicated in the lifetime maps for both the prepatterned (e) and the reference area (f). The prepatterned domains exhibit a larger area fill fraction of near-boundary crystallites with a higher carrier lifetime as compared to crystallites on the domain interior. The TRPL decays correspond to the average signal of 100 pixels.

single crystals, with one facet aligned to the substrate surface. ${ }^{33,38}$

A simplified classical nucleation and crystal growth model is described by the so-called LaMer curve, ${ }^{39}$ relating crystallization mechanisms to the solution concentration, which has also been applied to illustrate crystallization kinetics in perovskite films. ${ }^{28,40}$ The LaMer curve shows the concentration as a function of time, increasing because of solvent evaporation until the supersaturation limit for nucleation is reached. Once solutes are removed from the solution because of crystallization, the concentration decreases as a result. The absence of spontaneously nucleated domains, according to the LaMer model, between the Au-seeded domains in all prepatterned films indicates that the solution film in the prepatterned regions never reaches the supersaturation threshold required for spontaneous nucleation, $C_{\text {sup }}$, as indicated in the modified LaMer curve in Figure $1 \mathrm{~h}$. Instead, nucleation at the $\mathrm{Au}$ seeds occurs at a lower concentration, $C_{\mathrm{Au}}$. Although continued heating leads to sustained solvent evaporation, the simultaneous crystallization of the precursor molecules counteracts the increase in concentration, such that $C_{\text {sup }}$ is never reached before Au-seeded domain formation and crystallization are complete. This is discussed in more depth below.

As standard scanning electron microscopy (SEM) imaging is limited in identifying the crystalline nature of the domains, EBSD mapping was used to compare the micromorphology of prepatterned $\mathrm{MAPbI}_{3}$ domains with spontaneously nucleated films, by determining the spatial distribution of crystallites within the domains, their dimensions, and orientations (Figure $2 a-d)$. Figure $2 a$ shows the overlap of the image quality (brightness, IQ) with the inverse pole figure (color, IPF), representing the signal intensity and the crystal orientation obtained from fitting the Kikuchi lines (Figure 2c) to each pixel of the scanned area perpendicular to the substrate, respectively. Perpendicular to the substrate ( $z$-axis), EBSD reveals domains preferentially oriented along the (112) and (100) orientations, as confirmed by the X-ray diffraction (XRD) data shown in Figure 2e. The XRD data are indicative of the characteristic preferential crystal orientations (112) and (100), showing peaks at $20^{\circ}$ corresponding to the (112) and (200) reflections and at $40.5^{\circ}$ corresponding to the (224) and (400) reflections that are also observed in nonpatterned
$\mathrm{MAPbI}_{3}$ perovskite films ${ }^{37}$ annealed by FIRA and in orientationally pure crystalline films grown in a thermal gradient parallel to the substrate. ${ }^{41,42}$ EBSD mapping allows to distinguish individual crystallites by determining their inand out-of-plane crystal orientations, showing that crystallites close to the domain boundaries tend to be larger than those in the interior of the domains. This polycrystalline morphology of the domains with increasing crystallite sizes toward the domain boundaries, revealed by the EBSD measurements, indicates that a secondary heterogeneous nucleation process, originating from the crystal growth fronts at the substrate surface or the liquid-gas interface, gives rise to the characteristic crystal morphology of the domains. For completeness, the crystallographic orientations along $x$ - and $y$-directions are shown in Figure S2.

If the crystallite size depended only on their absolute distance to a nucleation site, increasing the nucleation site density would yield smaller domains and thus a smaller average crystallite size. However, the opposite effect is observed, where the larger boundary crystallites appear merely closer to the nucleation sites, indicating that the characteristic polycrystalline morphology is independent of the final domain size. Therefore, by increasing the nucleation density, the crystallite size distribution of the entire film is biased toward larger crystallite sizes.

The photoluminescence (PL) lifetime in perovskite films depends on the size of crystallites within a polycrystalline assembly. ${ }^{43}$ TRPL mapping was used to investigate whether the crystalline morphology within the domains correlates with the optoelectronic properties. In these experiments, the spatially resolved PL intensity and charge carrier lifetime $(\tau)$ within a domain of prepatterned perovskite films were compared to an unpatterned reference area without Au seeds (Figure 3). Both areas show a strong correlation of crystalline micromorphology with the PL intensity (Figure 3a,b) and $\tau$ maps (Figure $3 \mathrm{c}, \mathrm{d}$ ), indicating that micromorphology determines the luminescence and charge carrier dynamics. In the interior of the domains (position A in the PL maps), where the EBSD measurements show small crystallites, the lifetime is approximately 2 times shorter than that in larger crystallites near the domain boundaries (position B in the PL maps). Representative PL transients of these regions are illustrated in Figure $3 \mathrm{e}, \mathrm{f}$ for the prepatterned and reference areas, 

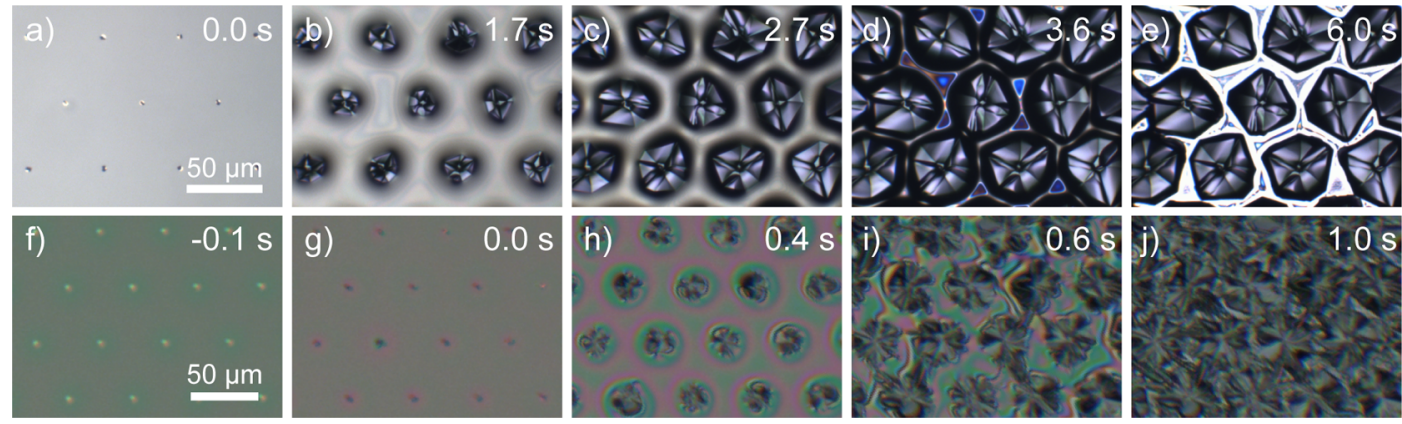

Figure 4. In situ observation of nucleation and crystallization of $\mathrm{MAPbI}_{3}$ films on an Au-patterned Si substrate heated to a constant temperature of $110{ }^{\circ} \mathrm{C}$. (a-e) Drop-cast; (f-j) spin-coated for $10 \mathrm{~s}$ at $4000 \mathrm{rpm}$ (as for FIRA); and subsequently annealed at $110{ }^{\circ} \mathrm{C}$ during in situ observation.

respectively. Both the patterned and reference areas exhibit very similar decays in each region of $\sim 5 \mathrm{~ns}$ in the interior (position A) of a prepatterned domain ( $6 \mathrm{~ns}$ for the reference) and $\sim 11$ ns near the boundaries (position B, 12 ns for the reference). Hence, we conclude that the characteristic micromorphology is preserved in Au-seeded prepatterned domains, and there is no evident modification of the PL characteristics by increasing the nucleation site density as compared to the reference area. By retaining this distribution of crystallite sizes within each domain, increasing the nucleation site density by patterning increases the proportion of large near-boundary crystallites that contribute to the overall longer PL lifetime of the entire perovskite film.

At the same time, seeding also has an effect on the morphology of the centers of the domains, that is, close to the nucleation site (Figure S3). The TRPL signal from the domain centers includes, however, a nonquantifiable contribution from the $\mathrm{Au}$ seed, making a TRPL comparison of the domain centers difficult. Unfortunately, because of the difference in roughness of the film between the center and the rest of the domain, EBSD mapping also was not able to resolve the crystallite dimensions at the domain centers. Figures S9 and S10 show exemplary atomic force microscopy (AFM) and cross-sectional SEM images, respectively. Note, however, that the topography and thickness of the films vary significantly with all the experimental parameters, such as Au array pitch, perovskite composition, annealing time, annealing temperature, and annealing method.

Note that the presence of Au seeds after crystallization could make this architecture impractical for use in solar cell devices because of the introduction of trap states and recombination sites as well as degradation processes because of $\mathrm{Au}$ migration. ${ }^{44,45}$ The deterministic nucleation effect of other metals (e.g., Pt and $\mathrm{Cu}$ ) was also tested, but their effect was not as consistent as that of $\mathrm{Au}$, whereas a nonmetallic material such as $\mathrm{SiO}_{2}$ did not show a nucleating effect at all (Figure S4). A material that is chemically and optoelectronically inert after crystallization but provides a similar nucleation catalysis effect would be an interesting candidate material for future integration into devices.

2.2. In Situ Observation of Nucleation and Crystallization. To further study the dynamic formation kinetics of the domain morphologies on patterned and unpatterned substrates, we observed the nucleation and crystallization processes in situ under an optical microscope ${ }^{34}$ using a heating stage to approximate the thermally induced solvent evaporation that drives the film crystallization in the FIRA experiments. Although FIRA is able to reach much higher peak temperatures and, therefore, shorter annealing times, we show that the hot-plate annealing achieves the same characteristic film morphology. Figure $4 a-e$ shows a sequence of snapshots corresponding to the different stages of nucleation and growth of $\mathrm{MAPbI}_{3}$ perovskite domains. The substrate was heated to a constant temperature under the optical microscope followed by drop-casting $2 \mu \mathrm{L}$ of a precursor solution onto the substrate. In each experiment, visible nucleation occurred simultaneously at all seed patches (within $0.14 \mathrm{~s}$ ). The growth rate, as defined by the area increase of the domains, was uniform, leading to regular hexagonal arrays of perovskite domains. Figure $4 \mathrm{f}-\mathrm{j}$ shows a similar in situ experiment, in which the precursor solution was spin-cast onto the substrate, similar to the FIRA fabrication protocol. In these spin-coated films, a polycrystalline micromorphology similar to that in FIRA films was obtained (SEM image in Figure S5). The thinner spin-cast solution film leads to an initially growing domain that reaches the liquid-air interface, inducing a secondary heterogeneous nucleation on the surface of the solution film, such that the domain grows in an alternating cascade of nucleation- and diffusion-driven growth.

The observations in the in situ experiments confirm the regularity of the domain patterns observed in the FIRA samples (Figure 1a-f). The nucleation at the Au seeds was simultaneous, and the crystallization growth rate from each $\mathrm{Au}$ patch (Figure 1h) was uniform, which leads to nearly perfect honeycomb patterns, seeded by the hexagonal Au lattice. In this seeded nucleation process, the saturation threshold for nucleation is sufficiently lowered, such that no domains originating from spontaneous nucleation were observed in the area occupied by the Au seed pattern.

Compared to spontaneously nucleated FIRA domains, the precursor solution adjacent to the nucleated crystal growth front is, therefore, not close to the supersaturation limit, $C_{\text {sup }}$ (Figure $1 \mathrm{~h}$ ), confirming the interpretation of the FIRA experiments (Figure $1 \mathrm{a}-\mathrm{f}$ ). Hence, secondary heterogeneous nucleation on the surface of the growing crystals is less frequent, resulting in fewer interruptions of growth of individual crystallites. This yields larger crystallites at the domain centers, relative to the domain interior, as shown in Figure S3.

In the case of spontaneous nucleation, the crystallization is overall faster because the solution is close to $C_{\text {sup }}$ in the entire film, hence, also adjacent to a growing crystal domain. This, on the one hand, leads to rapid crystal nucleation and growth, outpacing solute diffusion to the crystallites. Although providing good substrate coverage, this, on the other hand, 

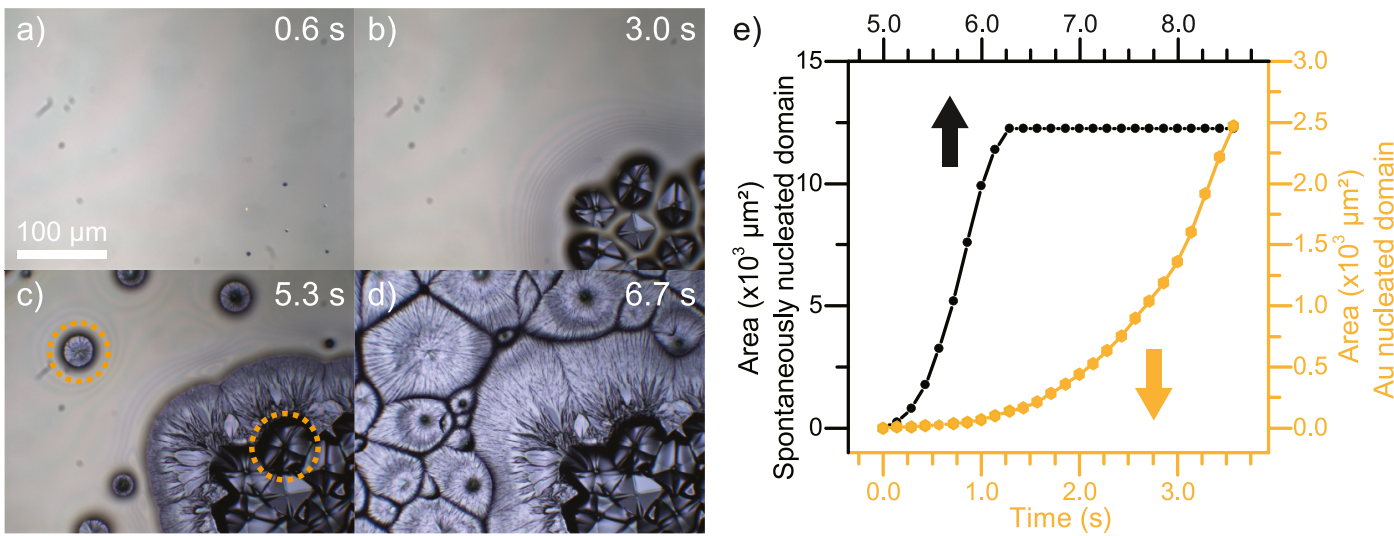

Figure 5. (a-d) $\mathrm{MAPbI}_{3}$ crystallizing on a patterned Si substrate heated to $110{ }^{\circ} \mathrm{C}$ from a drop-cast precursor solution. The images view a corner of a patterned array and a substrate region without nucleation seeds. (e) Measured area of an Au seed-nucleated and a spontaneously nucleated domain with respect to the first frame in which they were visible [indicated by dotted circles in (c)].
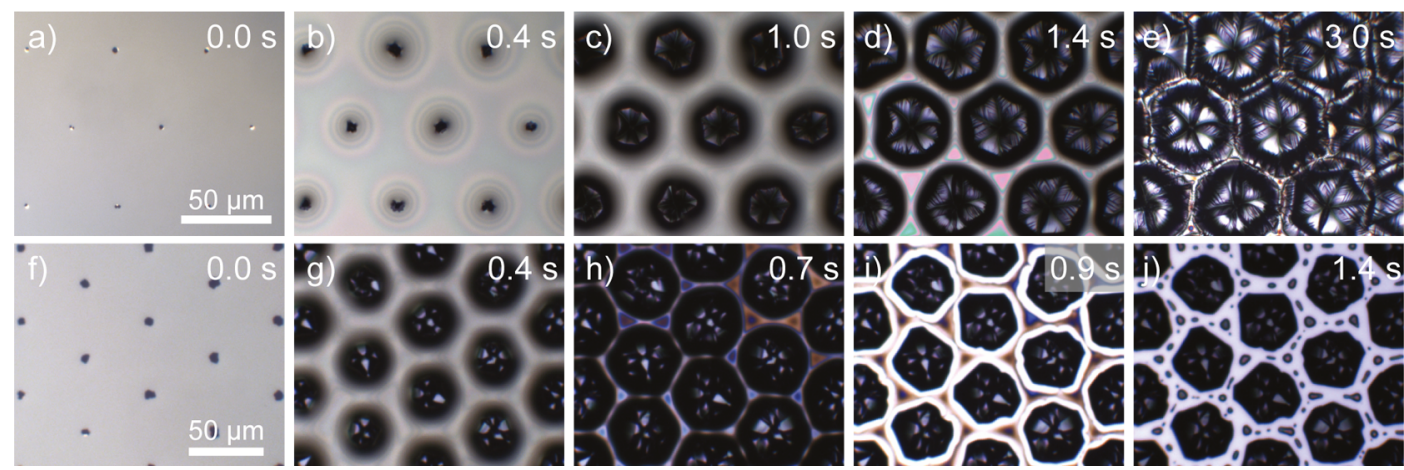

Figure 6. (a-e) Triple cation perovskite, $\mathrm{Cs}_{0.05}\left(\mathrm{MA}_{0.17} \mathrm{FA}_{0.83}\right)_{0.95} \mathrm{~Pb}\left(\mathrm{I}_{0.83} \mathrm{Br}_{0.17}\right)_{3}$, nucleating and crystallizing on an Au-patterned Si substrate from a drop-cast precursor solution on a hot plate set to a constant temperature of $110{ }^{\circ} \mathrm{C}$. $(\mathrm{f}-\mathrm{j}) \mathrm{MAPbI}_{3}$ perovskite nucleation and growth from a pure DMF-based precursor solution, drop-cast onto an Au-patterned Si substrate at $110{ }^{\circ} \mathrm{C}$.

results in small individual crystallites that form in a series of interrupted nucleation and growth processes.

The interplay of solute diffusion and crystal growth rate also affects the substrate coverage in the Au-nucleated case (see Figure $1 \mathrm{~g}$ ). Lowering the saturation threshold of nucleation reduces the initial rate of crystal growth, and thus the diffusion of solutes is relatively faster, leading to larger crystallites but also a higher degree of material accumulation close to the nucleation site (i.e., locally thicker perovskite domains). Figure $5 \mathrm{a}-\mathrm{d}$ shows a direct comparison of Au-seeded and spontaneous nucleation and subsequent diffusion-driven crystal growth of the domains in the same film. The time offset between the nucleation types correlates to the offset in supersaturation threshold values, $C_{\text {sup }}$ and $C_{\mathrm{Au}}$ as the solvent evaporates over time (Figure $1 \mathrm{~h}$ ). The images also show that the absence of spontaneous nucleation in the prepatterned areas, as discussed above, does not only arise from precursor depletion near the growing seeded crystals but also from the mentioned time offset because of different critical concentrations for the respective nucleation types. In the region beyond the Au-seeded pattern, spontaneous nucleation only occurs once the solution reaches $C_{\text {sup }}$, when the Au-seeded domains have already reached their maximum extent.

The difference in the crystal growth rate is shown in Figure 5e. Both for seeded and spontaneous nucleation, the final size of the domains is determined by the adjacently grown domains, that is, by the Voronoi tessellation of all nucleation sites. Spontaneous nucleation occurs for a more supersaturated solution compared to seeded nucleation, and their rapid growth yields a different micromorphology.

The Au-seeded nucleation leads to diffusion-driven domain growth, whereas the spontaneously nucleated domain growth is characterized by a more rapid interplay between the diffusion-driven crystal growth and heterogeneous secondary nucleation of the highly supersaturated precursor solution near the surface of the crystal growth front. This growth mechanism leads to a starlike polycrystalline micromorphology similar to the morphology in $\mathrm{MAPbI}_{3}$ perovskite films annealed by FIRA. ${ }^{28,37}$

Seeding the nucleation of perovskite domains at low solution concentrations during solvent evaporation, thus, enables an improved control over the polycrystalline morphology in single-step rapid thermally annealed perovskite films. The effect of lowering the saturation threshold can, however, lead to disconnected domains because of material depletion between the growing domains, which is governed by an interplay of the crystal growth rate relative to the diffusion of the solutes and the distance between nucleation seeds. Welldefined disconnected perovskite domains may on the one hand be useful for some applications, ${ }^{46}$ particularly in the case of cubic $\mathrm{MAPbBr}_{3}$ that forms faceted isolated domains (Figure $1 d-f)$. If, on the other hand, a uniform substrate coverage is desired, the nucleation site density and the annealing temperature have to be appropriately adjusted.

Importantly, Au-seeded patterning also works for a range of perovskite compositions such as the high band gap $\mathrm{MAPbBr}_{3}$ 
(Figure $1 \mathrm{~d}-\mathrm{f}$ ), the widely used triple cation $\mathrm{Cs}_{0.05}\left(\mathrm{MA}_{0.17} \mathrm{FA}_{0.83}\right)_{0.95} \mathrm{~Pb}\left(\mathrm{I}_{0.83} \mathrm{Br}_{0.17}\right)_{3}$ (Figure 6a-e), and MA-free $\mathrm{Cs}_{0.15} \mathrm{FA}_{0.85} \mathrm{PbI}_{3}$ (Figure S6).

In particular, the triple cation and dimethylformamide (DMF)-based $\mathrm{MAPbI}_{3}$ offer further insight into the morphology formation in the single-step rapid thermally annealed perovskite films. At low growth speeds parallel to the substrate, pattern formation is aided by surface tension and convection effect, also observed in domain formation in the meniscus coating methods of perovskites. ${ }^{47}$ The thin-film interference visible in the optical microscopy images in Figures 4 and 6 between the growing domains shows that the thickness of the solution film varies between the growing domains. This effect is strongest in the pure DMF-based $\mathrm{MAPbI}_{3}$ depicted in Figure $6 \mathrm{f}-\mathrm{j}$, where dewetting of the solution film produces secondary and tertiary lattices of small domains. This ripple effect is due to a complex interplay of solution flow, solute diffusion, the solvent evaporation, and the crystal growth. ${ }^{48,49}$

This effect depends strongly on the crystal growth kinetics and, thus, on both the perovskite composition and the solvent mixture. The pure DMF-based $\mathrm{MAPbI}_{3}$ precursor solution lacks the crystallization retardation afforded by dimethylsulfoxide (DMSO), and the material accumulates more strongly close to the nucleation sites.

In the meniscus-assisted solution printing of perovskites, $\mathrm{He}$ et al. found an initially high crystal growth rate after nucleation, causing the domains to grow quadratically with time (corresponding to linear radial growth), and a second slower phase with a linear area growth caused by an overlap of the solute diffusion areas of several growing crystals. ${ }^{30}$ Figure 5e shows an initial high crystal growth rate after nucleation in the in situ observation because of continued solvent evaporation. The growth rate reaches an inflection point when the diffusion areas of adjacently growing domains overlap, slowing down the crystal growth until the domains meet, forming their mutual boundaries. The change in growth rate corresponds to the observation of varying crystallite sizes within the domains, regardless of the domain size. Thus, the crystallite size within the micromorphology of the domains is determined by the crystal growth rate rather than the absolute distance to the nucleation site.

DMSO plays an important role in affecting the crystal growth rate. It strongly coordinates with $\mathrm{PbI}_{6}$ molecules, forming a metastable intermediate phase in the crystallization process of $\mathrm{MAPbI}_{3}$, thus retarding the crystallization process. $^{50-52}$ To understand its effect in the crystallization process, we repeated the in situ experiment with a precursor solution of $\mathrm{MAPbI}_{3}$ in pure DMF. Figure $6 \mathrm{~b}$ shows that, without the DMSO-mediated metastable phase, crystal growth parallel to the substrate is slowed down, suggesting that the intermediate phase is critical in achieving uniform compact films.

Moreover, at equal temperatures, the $\mathrm{Cs}_{0.05}\left(\mathrm{MA}_{0.17} \mathrm{FA}_{0.83}\right)_{0.95} \mathrm{~Pb}\left(\mathrm{I}_{0.83} \mathrm{Br}_{0.17}\right)_{3}$ composition achieves more uniform substrate coverage compared to $\mathrm{MAPbI}_{3}$ (see Figure $6 a$ ), suggesting that the high efficiency and stability of this perovskite composition do not only arise from its chemical composition but also that the heterogeneity in its components prevents a highly ordered faceted crystal growth immediately after nucleation.

Spatial heterogeneity in triple-cation perovskite films observed by Bercegol et al. suggests that Cs doping may facilitate favorable morphology evolution during crystallization rather than optoelectronic effects because of its presence in the crystallized perovskite film. ${ }^{53,54}$ Figure S7 shows that the XRD measurement of a triple cation perovskite film annealed with FIRA is in good agreement with a reference film prepared with the antisolvent method. This is in contrast to a comparable $\mathrm{MAPbI}_{3}$ study, which exhibits characteristic preferential crystal orientations after rapid thermal annealing (e.g., FIRA) but not when using the antisolvent method (Figure $2 \mathrm{e}$ ).

In contrast, the highly symmetric cubic crystal lattice of $\mathrm{MAPbBr}_{3}{ }^{38}$ yields isolated highly faceted domains that appear to consist of only a few individual single crystals (see Figure $1 \mathrm{~d}-\mathrm{f}$ ). Hence, although the consistent $\mathrm{MAPbBr}_{3}$ pattern morphology shows that the saturation threshold for nucleation at the $\mathrm{Au}$ seeds is lowered similar to that of $\mathrm{MAPbI}_{3}$, the growth rate has to be significantly higher to achieve completely isolated platelets under the same experimental conditions that yield a complete substrate coverage by a $\mathrm{MAPbI}_{3}$ film.

\section{CONCLUSIONS}

Using prepatterned substrates, the nucleation of domains was controlled with $\mathrm{Au}$ seeds in perovskite films annealed by FIRA. The seeding lowers the nucleation threshold, enabling an otherwise inaccessible regime in the kinetic space of nucleation and crystal growth. The modified nucleation site density and lowering of the nucleation threshold led to morphological differences that affect the micromorphology of the polycrystalline perovskite films and their optoelectronic properties. It was shown that the crystallite size in the domains correlates with the crystal growth rate and solute concentration at the crystal growth front of growing domains. This result is important as it allows to engineer the crystallite size in nucleated perovskite films, allowing a systematic study of the electronic effects of grain boundaries, which in the long run will enable to improve the performance and lifetime of devices.

Although Au seeds are reliable nucleation catalysts and are easily patterned onto substrates, they have the evident caveats in an optoelectronic device, introducing trap states and recombination sites and contributing to faster degradation mechanisms. In the case where geometric patterning is not desired but seeding-assisted morphology control of compact films is the objective, other seeding particles as additives such as quantum dots are beginning to be explored ${ }^{55,56}$ and should be of interest, especially in fabrication upscaling with singlestep thermal annealing methods.

The spatial predetermination of the nucleation sites enables a detailed local study of the nucleation and crystal growth processes, as demonstrated in the in situ experiments. Knowing the location of the nucleation site and the direction and scope of crystal growth a priori enables the locally targeted study of the evolution from the precursor solution to perovskite crystal beyond the visual observation with the optical microscope.

Beyond PSCs, the deterministic patterning of isolated perovskite domains can be of interest for other applications of perovskite materials such as sensing, light emission (e.g., LEDs and lasers), and light detection. Further optimizing the seeding and annealing parameters with a device application in mind, obtaining large-scale perovskite arrays with near-single crystal morphologies, could be possible, similar to recent developments but compatible with the FIRA method. ${ }^{11}$ This approach should be compatible with the thin-film transfer methods such as template stripping, for example, for applications requiring substrates that are incompatible with the growth of high-quality perovskite films. ${ }^{57}$ 


\section{EXPERIMENTAL SECTION}

4.1. Substrate Patterning. A photoresist layer (ECI 3007, MicroChemicals) was spin-coated (Süss ACS200 Gen3) onto siliconor ITO sputter-coated (500 nm, Pfeiffer SPIDER 600) glass wafers. Hexagonal arrays of discs with $3 \mu \mathrm{m}$ diameter and of varying piths were exposed by a laser writer (405 nm, Heidelberg MLA150) and developed (AZ $726 \mathrm{MIF}$, MicroChemicals). The wafers were then coated with a $1 \mathrm{~nm} \mathrm{Cr}$ and a $15 \mathrm{~nm}$ Au layer by thermal evaporation (Minilab-80, Moorfield Nanotechnology), followed by a standard liftoff step (Remover 1165, Microposit), rinsing in IPA and $\mathrm{H}_{2} \mathrm{O}$, and a final blow dry with $\mathrm{N}_{2}$. The wafers were then diced into $12 \times 12 \mathrm{~mm}^{2}$ samples using a diamond scribe.

4.2. Perovskite Synthesis. The lead iodide $\left(\mathrm{PbI}_{2}\right)$ and lead bromide $\left(\mathrm{PbBr}_{2}\right)$ precursors were purchased from TCI; the organic salts methylammonium iodide (MAI), methylammonium bromide $(\mathrm{MABr})$, formamidinium iodide (FAI), and formamidinium bromide (FABr) from Greatcell Solar Materials; and cesium iodide (CsI), anhydrous DMF, and anhydrous DMSO from Sigma-Aldrich.

The methylammonium lead tri-iodide $\left(\mathrm{MAPbI}_{3}\right)$ and methylammonium lead tribromide $\left(\mathrm{MAPbBr}_{3}\right)$ precursor solutions were prepared by mixing a 1:1 ratio of $\mathrm{MAI}$ and $\mathrm{PbI}_{2}$ and $\mathrm{MABr}$ and $\mathrm{PbBr}_{2}$ respectively, in a $3: 1 \mathrm{v} / \mathrm{v}$ DMF/DMSO solvent mixture or in pure DMF to obtain a concentration of $1.2 \mathrm{M}$. The triple cation $\left(\mathrm{Cs}_{0.05}\left(\mathrm{MA}_{0.17} \mathrm{FA}_{0.83}\right)_{0.95} \mathrm{~Pb}\left(\mathrm{I}_{0.83} \mathrm{Br}_{0.17}\right)_{3}\right)$ precursor solution was obtained from a $83: 17 \mathrm{v} / \mathrm{v}$ ratio of $1.24 \mathrm{M}$ formamidinium lead tri-iodide $\left(\mathrm{FAPbI}_{3}\right)$ and $\mathrm{MAPbBr}_{3}$ solutions, with an overstoichiometric ratio of 1:1.09 of both $\mathrm{MABr} / \mathrm{PbBr}_{2}$ and $\mathrm{FAI} / \mathrm{PbI}_{2}$, prepared in $4: 1 \mathrm{v} / \mathrm{v}$ DMF/DMSO. For the $\mathrm{Cs}_{0.15} \mathrm{FA}_{0.85} \mathrm{PbI}_{3}$ perovskite, a $1.24 \mathrm{M} 1: 1.09$ overstoichiometric $\mathrm{FAPbI}_{3}$ solution was mixed with CsI in $4: 1 \mathrm{v} / \mathrm{v}$ DMF/DMSO. The precursor solution preparation was carried out in a glovebox under a $\mathrm{N}_{2}$ atmosphere.

The samples, using the FIRA method, were prepared by spincoating the precursor solution in a single step at $4000 \mathrm{rpm}$ for $10 \mathrm{~s}$ onto patterned $\mathrm{Si}$ or ITO-coated glass substrates (after $5 \mathrm{~min} \mathrm{O}_{2}$ plasma etching), then irradiated for $1.4 \mathrm{~s}$ in the FIRA chamber, and kept inside the chamber for an additional $20 \mathrm{~s}$. The entire process was carried out in a glovebox under a $\mathrm{N}_{2}$ atmosphere.

4.3. Microscopy and Structural Characterization. For in situ microscopy observations, the patterned silicon substrates were initially exposed to a $5 \mathrm{~min} \mathrm{O}_{2}$ plasma step and then kept at a constant elevated temperature on a Linkam HFS350X temperature-controlled stage installed under a ZEISS Axio Scope.A1 optical microscope equipped with a Point Grey Grasshopper3 camera using a $20 \times$ objective. Unless otherwise indicated, $2 \mu \mathrm{L}$ of the respective precursor solution was drop-cast onto the heated substrate. The in situ observations were limited to patterned Si substrates, as the Au patches on ITO-coated glass substrates heat more strongly relative to the substrate than on $\mathrm{Si}$, causing dewetting from these patches. In this case, perovskite nucleation occurs at the boundaries of the dewetted areas (Figure S8).

XRD measurements on the films were performed using a Rigaku Ultima-IV diffractometer using $\mathrm{Cu} \mathrm{K} \alpha$ radiation.

SEM images were obtained with a Tescan Mira3 LM FE SEM system, with a field emission source operated at an acceleration voltage of $5 \mathrm{kV}$ using an Everhart-Thornley-type secondary electron detector.

For EBSD measurements, a direct-electron detector was used. A detailed description of the setup, data collection and procedure for fitting the Hough's space is reported in Muscarella et al. ${ }^{37} 7 \mathrm{kV}, 100$ $\mathrm{pA}, 50 \mathrm{~ms}, 12.4 \mathrm{~mm}, 500 \mathrm{~nm}$ were used as voltage, current, exposure time, working distance and step size of the measurement, respectively. These scanning parameters correspond to a $5 \mathrm{nA}$ ms electron dose per pixel. The collected Kikuchi patterns were indexed using the space group I4/ $\mathrm{mcm}$ (tetragonal symmetry).

The TRPL mapping system is based on PicoQuant systems composed by a FluoTime 300 unit and a microscope system (MicroTime 100). For excitation, a $639 \mathrm{~nm}$ laser with $\sim 100 \mathrm{ps}$ pulse width with a frequency of $3 \mathrm{MHz}$ has been used. The beam diameter was roughly $130 \mu \mathrm{m}$ (13.5\% metric), as measured with a NanoScan2 beam profiler. The excitation photon density was $\sim 2 \times 1012$ photons $/ \mathrm{cm}^{2} /$ pulse. To collect the emitted light, an Olympus longrange $20 \times$ objective (LCPLN20XIR) with a numerical aperture of 0.45 was used. The emitted light is guided to a detection fiber of 50 $\mu \mathrm{m}$ acting as a pinhole, which is coupled to the FluoTime unit including the input optics, to focus the light into the monochromator and the photomultiplier detector (PMA 192). A Time-Harp 260 Nano TCPSC card in long-range mode has been used for photon counting. A wide-range scanner stage from Physik Instrumente is used (M687.7) for mapping. The dwell time was set to $5 \mathrm{~ms} /$ decay with a step size of $\sim 0.5 \mu \mathrm{m} / \mathrm{pixel}$. The system has a global illumination local detection configuration (wide-field) with an optical resolution of $\sim 2$ $\mu \mathrm{m}$. A single exponential equation has been used to fit the decay at each pixel. The samples used for TRPL correspond to a layer structure glass/ITO/local Au seeds/ $/ \mathrm{MAPbI}_{3}$.

\section{ASSOCIATED CONTENT}

\section{Supporting Information}

The Supporting Information is available free of charge at https://pubs.acs.org/doi/10.1021/acsami.0c20958.

$50 \mu \mathrm{m}$ pitch $\mathrm{MAPbBr}_{3}$; domain centers; platinum and copper nucleation seeds; final in situ film; EBSD in-plane orientations; optical microscopy of in situ crystallization of MA-free perovskite; XRD of triple cation FIRA and antisolvent; SEM image of $\mathrm{MAPbBr}_{3}$ on a Au-patterned ITO substrate; cross section of patterned $\mathrm{MAPbI}_{3}$; and AFM image of patterned $\mathrm{MAPbI}_{3}$ (PDF)

\section{AUTHOR INFORMATION}

\section{Corresponding Authors}

Michael Saliba - Institute for Photovoltaics (IPV), University of Stuttgart, D-70569 Stuttgart, Germany; Helmholtz Young Investigator Group FRONTRUNNER, IEK5-Photovoltaik, Forschungszentrum, Jülich D-52425, Germany; 10 orcid.org/ 0000-0002-6818-9781; Email: michael.saliba@ipv.unistuttgart.de

Ullrich Steiner - Adolphe Merkle Institute, University of Fribourg, 1700 Fribourg, Switzerland; ㅇo․ orid.org/00000001-5936-339X; Email: ullrich.steiner@unifr.ch

\section{Authors}

Antonio Günzler - Adolphe Merkle Institute, University of Fribourg, 1700 Fribourg, Switzerland

Esteban Bermúdez-Ureña - Adolphe Merkle Institute, University of Fribourg, 1700 Fribourg, Switzerland; () orcid.org/0000-0002-8964-9660

Loreta A. Muscarella - Center for Nanophotonics, AMOLF, 1098 XG Amsterdam, The Netherlands; (1) orcid.org/00000002-0559-4085

Mario Ochoa - Laboratory for Thin-Films and Photovoltaics, Empa-Swiss Federal Laboratories for Materials Science and Technology, 8600 Duebendorf, Switzerland

Efraín Ochoa-Martínez - Adolphe Merkle Institute, University of Fribourg, 1700 Fribourg, Switzerland

Bruno Ehrler - Center for Nanophotonics, AMOLF, 1098 XG Amsterdam, The Netherlands

Complete contact information is available at:

https://pubs.acs.org/10.1021/acsami.0c20958

\section{Notes}

The authors declare no competing financial interest. 


\section{ACKNOWLEDGMENTS}

This work was partially supported by the Swiss National Science Foundation (SNSF) through grants numbers 153990 and 186453. A.G., E.B.-U., E.O.-M., and U.S. acknowledge the financial support by the Adolphe Merkle Foundation. The work of L.A.M. and B.E. is part of the Dutch Research Council (NWO) and was performed at the research institute, AMOLF. The work of L.A.M. was supported by NWO Vidi grant 016.Vidi.179.005. M.O. acknowledges financial support partially from the Swiss State Secretary for Education, Research, and Innovation (SERI) under contract number 17.00105 (EMPIR project HyMet). The EMPIR program is co-financed by the participating states and by the European Union's Horizon 2020 research and innovation program. E.O.-M. acknowledges support from the Marie Skłodowska Curie fellowship, H2020 grant agreement no. 841005 .

\section{REFERENCES}

(1) Green, M.; Dunlop, E.; Hohl-Ebinger, J.; Yoshita, M.; Kopidakis, N.; Hao, X. Solar Cell Efficiency Tables (Version 57). Prog. Photovoltaics 2021, 29, 3-15.

(2) Jeon, N. J.; Noh, J. H.; Yang, W. S.; Kim, Y. C.; Ryu, S.; Seo, J.; Seok, S. I. Compositional Engineering of Perovskite Materials for High-Performance Solar Cells. Nature 2015, 517, 476-480.

(3) Saliba, M.; Matsui, T.; Seo, J.-Y.; Domanski, K.; Correa-Baena, J.-P.; Nazeeruddin, M. K.; Zakeeruddin, S. M.; Tress, W.; Abate, A.; Hagfeldt, A.; Grätzel, M. Cesium-Containing Triple Cation Perovskite Solar Cells: Improved Stability, Reproducibility and High Efficiency. Energy Environ. Sci. 2016, 9, 1989-1997.

(4) National Renewable Energy Laboratory. Best Research-Cell Efficiency Chart. https://www.nrel.gov/pv/assets/pdfs/best-researchcell-efficiencies.20200708.pdf (accessed Jan 2021).

(5) Pazos-Outón, L. M.; Xiao, T. P.; Yablonovitch, E. Fundamental Efficiency Limit of Lead Iodide Perovskite Solar Cells. J. Phys. Chem. Lett. 2018, 9, 1703-1711.

(6) Shockley, W.; Queisser, H. J. Detailed Balance Limit of Efficiency of p-n Junction Solar Cells. J. Appl. Phys. 1961, 32, 510519.

(7) Hu, H.; Singh, M.; Wan, X.; Tang, J.; Chu, C.-W.; Li, G. Nucleation and Crystal Growth Control for Scalable SolutionProcessed Organic-Inorganic Hybrid Perovskite Solar Cells. J. Mater. Chem. A 2020, 8, 1578-1603.

(8) Wang, F.; Cao, Y.; Chen, C.; Chen, Q.; Wu, X.; Li, X.; Qin, T.; Huang, W. Materials toward the Upscaling of Perovskite Solar Cells: Progress, Challenges, and Strategies. Adv. Funct. Mater. 2018, 28, 1803753.

(9) Murali, B.; Kolli, H. K.; Yin, J.; Ketavath, R.; Bakr, O. M.; Mohammed, O. F. Single Crystals: The Next Big Wave of Perovskite Optoelectronics. ACS Mater. Lett. 2020, 2, 184-214.

(10) Chen, Z.; Turedi, B.; Alsalloum, A. Y.; Yang, C.; Zheng, X.; Gereige, I.; Alsaggaf, A.; Mohammed, O. F.; Bakr, O. M. SingleCrystal MAPbI3 Perovskite Solar Cells Exceeding 21\% Power Conversion Efficiency. ACS Energy Lett. 2019, 4, 1258-1259.

(11) Lei, Y.; Chen, Y.; Zhang, R.; Li, Y.; Yan, Q.; Lee, S.; Yu, Y.; Tsai, H.; Choi, W.; Wang, K.; Luo, Y.; Gu, Y.; Zheng, X.; Wang, C.; Wang, C.; Hu, H.; Li, Y.; Qi, B.; Lin, M.; Zhang, Z.; Dayeh, S. A.; Pharr, M.; Fenning, D. P.; Lo, Y.-H.; Luo, J.; Yang, K.; Yoo, J.; Nie, W.; Xu, S. A Fabrication Process for Flexible Single-crystal Perovskite Devices. Nature 2020, 583, 790-795.

(12) Lee, L.; Baek, J.; Park, K. S.; Lee, Y. E. K.; Shrestha, N. K.; Sung, M. M. Wafer-Scale Single-Crystal Perovskite Patterned Thin Films Based on Geometrically-Confined Lateral Crystal Growth. Nat. Commun. 2017, 8, 15882.

(13) Kayes, B. M.; Nie, H.; Twist, R.; Spruytte, S. G.; Reinhardt, F.; Kizilyalli, I. C.; Higashi, G. S. $27.6 \%$ Conversion Efficiency, A New Record for Single-Junction Solar Cells Under 1 Sun Illumination.
Conference Record of the IEEE Photovoltaic Specialists Conference, 2011; pp 4-8.

(14) Metaferia, W.; Schulte, K. L.; Simon, J.; Johnston, S.; Ptak, A. J. Gallium Arsenide Solar Cells Grown at Rates Exceeding $300 \mu \mathrm{m} \mathrm{h}^{-1}$ by Hydride Vapor Phase Epitaxy. Nat. Commun. 2019, 10, 3361.

(15) Tailor, N. K.; Abdi-Jalebi, M.; Gupta, V.; Hu, H.; Dar, M. I.; Li, G.; Satapathi, S. Recent Progress in Morphology Optimization in Perovskite Solar Cell. J. Mater. Chem. A 2020, 8, 21356-21386.

(16) Li, Y.; Ji, L.; Liu, R.; Zhang, C.; Mak, C. H.; Zou, X.; Shen, H.H.; Leu, S.-Y.; Hsu, H.-Y. A Review on Morphology Engineering for Highly Efficient and Stable Hybrid Perovskite Solar Cells. J. Mater. Chem. A 2018, 6, 12842-12875.

(17) Zhang, W.; Saliba, M.; Moore, D. T.; Pathak, S. K.; Hörantner, M. T.; Stergiopoulos, T.; Stranks, S. D.; Eperon, G. E.; AlexanderWebber, J. A.; Abate, A.; Sadhanala, A.; Yao, S.; Chen, Y.; Friend, R. H.; Estroff, L. A.; Wiesner, U.; Snaith, H. J. Ultrasmooth OrganicInorganic Perovskite Thin-Film Formation and Crystallization for Efficient Planar Heterojunction Solar Cells. Nat. Commun. 2015, 6, 6142.

(18) Steirer, K. X.; Schulz, P.; Teeter, G.; Stevanovic, V.; Yang, M.; Zhu, K.; Berry, J. J. Defect Tolerance in Methylammonium Lead Triiodide Perovskite. ACS Energy Lett. 2016, 1, 360-366.

(19) Lee, J.-W.; Bae, S.-H.; De Marco, N.; Hsieh, Y.-T.; Dai, Z.; Yang, Y. The Role of Grain Boundaries in Perovskite Solar Cells. Mater. Today Energy 2018, 7, 149-160.

(20) Foley, B. J.; Girard, J.; Sorenson, B. A.; Chen, A. Z.; Scott Niezgoda, J.; Alpert, M. R.; Harper, A. F.; Smilgies, D.-M.; Clancy, P.; Saidi, W. A.; Choi, J. J. Controlling Nucleation, Growth, and Orientation of Metal Halide Perovskite Thin Films with Rationally Selected Additives. J. Mater. Chem. A 2017, 5, 113-123.

(21) Pham, N. D.; Tiong, V. T.; Yao, D.; Martens, W.; Guerrero, A.; Bisquert, J.; Wang, H. Guanidinium Thiocyanate Selective Ostwald Ripening Induced Large Grain for High Performance Perovskite Solar Cells. Nano Energy 2017, 41, 476-487.

(22) Bae, S.; Jo, J. W.; Lee, P.; Ko, M. J. Controlling the Morphology of Organic-Inorganic Hybrid Perovskites through Dual AdditiveMediated Crystallization for Solar Cell Applications. ACS Appl. Mater. Interfaces 2019, 11, 17452-17458.

(23) Wang, F.; Shimazaki, A.; Yang, F.; Kanahashi, K.; Matsuki, K.; Miyauchi, Y.; Takenobu, T.; Wakamiya, A.; Murata, Y.; Matsuda, K. Highly Efficient and Stable Perovskite Solar Cells by Interfacial Engineering Using Solution-Processed Polymer Layer. J. Phys. Chem. C 2017, 121, 1562-1568.

(24) Roose, B.; Dey, K.; Chiang, Y.-H.; Friend, R. H.; Stranks, S. D. A Critical Assessment of the Use of Excess Lead Iodide in Lead Halide Perovskite Solar Cells. J. Phys. Chem. Lett. 2020, 11, 65056512.

(25) Fu, Y.; Zhu, H.; Chen, J.; Hautzinger, M. P.; Zhu, X.-Y.; Jin, S. Metal Halide Perovskite Nanostructures for Optoelectronic Applications and the Study of Physical Properties. Nat. Rev. Mater. 2019, 4, $169-188$.

(26) Watson, B. L.; Rolston, N.; Printz, A. D.; Dauskardt, R. H. Scaffold-Reinforced Perovskite Compound Solar Cells. Energy Environ. Sci. 2017, 10, 2500-2508.

(27) Yang, X.; Wu, J.; Liu, T.; Zhu, R. Patterned Perovskites for Optoelectronic Applications. Small Methods 2018, 2, 1800110.

(28) Sanchez, S.; Hua, X.; Phung, N.; Steiner, U.; Abate, A. Flash Infrared Annealing for Antisolvent-Free Highly Efficient Perovskite Solar Cells. Adv. Energy Mater. 2018, 8, 1702915.

(29) Sánchez, S.; Vallés-Pelarda, M.; Alberola-Borràs, J.-A.; Vidal, R.; Jerónimo-Rendón, J. J.; Saliba, M.; Boix, P. P.; Mora-Seró, I. Flash Infrared Annealing as a Cost-Effective and Low Environmental Impact Processing Method for Planar Perovskite Solar Cells. Mater. Today 2019, 31, 39-46.

(30) He, M.; Li, B.; Cui, X.; Jiang, B.; He, Y.; Chen, Y.; O’Neil, D.; Szymanski, P.; Ei-Sayed, M. A.; Huang, J.; Lin, Z. Meniscus-Assisted Solution Printing of Large-Grained Perovskite Films for HighEfficiency Solar Cells. Nat. Commun. 2017, 8, 16045. 
(31) Sanchez, S.; Steiner, U.; Hua, X. Phase Evolution during Perovskite Formation - Insight from Pair Distribution Function Analysis. Chem. Mater. 2019, 31, 3498-3506.

(32) Sánchez, S.; Hua, X.; Günzler, A.; Bermúdez-Ureña, E.; Septiadi, D.; Saliba, M.; Steiner, U. Flash Infrared Pulse Time Control of Perovskite Crystal Nucleation and Growth from Solution. Cryst. Growth Des. 2020, 20, 670-679.

(33) Geske, T.; Li, J.; Worden, M.; Shan, X.; Chen, M.; Bade, S. G. R.; Yu, Z. Deterministic Nucleation for Halide Perovskite Thin Films with Large and Uniform Grains. Adv. Funct. Mater. 2017, 27, 1702180.

(34) Li, Y.; Zhi, L.; Ge, G.; Zhao, Z.; Cao, X.; Chen, F.; Cui, X.; Lin, F.; Ci, L.; Sun, J.; Zhuang, D.; Wei, J. Investigation on Crystallization of $\mathrm{CH}_{3} \mathrm{NH}_{3} \mathrm{PbI}_{3}$ Perovskite and its Intermediate Phase from Polar Aprotic Solvents. Cryst. Growth Des. 2019, 19, 959-965.

(35) Ash, P. F.; Bolker, E. D. Generalized Dirichlet Tessellations. Geometriae Dedicata 1986, 20, 209-243.

(36) Dunlap-Shohl, W. A.; Zhou, Y.; Padture, N. P.; Mitzi, D. B. Synthetic Approaches for Halide Perovskite Thin Films. Chem. Rev. 2019, 119, 3193-3295.

(37) Muscarella, L. A.; Hutter, E. M.; Sanchez, S.; Dieleman, C. D.; Savenije, T. J.; Hagfeldt, A.; Saliba, M.; Ehrler, B. Crystal Orientation and Grain Size: Do They Determine Optoelectronic Properties of $\mathrm{MAPbI}_{3}$ Perovskite? J. Phys. Chem. Lett. 2019, 10, 6010-6018.

(38) Chen, F.; Zhu, C.; Xu, C.; Fan, P.; Qin, F.; Gowri Manohari, A.; Lu, J.; Shi, Z.; Xu, Q.; Pan, A. Crystal Structure and Electron Transition Underlying Photoluminescence of Methylammonium Lead Bromide Perovskites. J. Mater. Chem. C 2017, 5, 7739-7745.

(39) Lamer, V. K.; Dinegar, R. H. Theory, Production and Mechanism of Formation of Monodispersed Hydrosols. J. Am. Chem. Soc. 1950, 72, 4847-4854.

(40) Ding, B.; Li, Y.; Huang, S. Y.; Chu, Q. Q.; Li, C. X.; Li, C. J.; Yang, G. J. Material Nucleation/Growth Competition Tuning towards Highly Reproducible Planar Perovskite Solar Cells with Efficiency Exceeding 20\%. J. Mater. Chem. A 2017, 5, 6840-6848.

(41) Cho, N.; Li, F.; Turedi, B.; Sinatra, L.; Sarmah, S. P.; Parida, M. R.; Saidaminov, M. I.; Murali, B.; Burlakov, V. M.; Goriely, A.; Mohammed, O. F.; Wu, T.; Bakr, O. M. Pure Crystal Orientation and Anisotropic Charge Transport in Large-Area Hybrid Perovskite Films. Nat. Commun. 2016, 7, 13407.

(42) Hasan, M. M.; Clegg, C.; Manning, M.; El Ghanam, A.; Su, C.; Harding, M. D.; Bennett, C.; Hill, I. G.; Koleilat, G. I. Stable Efficient Methylammonium Lead Iodide Thin Film Photodetectors with Highly Oriented Millimeter-Sized Crystal Grains. ACS Photonics 2020, 7, 57-67.

(43) D’Innocenzo, V.; Srimath Kandada, A. R.; De Bastiani, M.; Gandini, M.; Petrozza, A. Tuning the Light Emission Properties by Band Gap Engineering in Hybrid Lead Halide Perovskite. J. Am. Chem. Soc. 2014, 136, 17730-17733.

(44) Domanski, K.; Correa-Baena, J.-P.; Mine, N.; Nazeeruddin, M. K.; Abate, A.; Saliba, M.; Tress, W.; Hagfeldt, A.; Grätzel, M. Not All That Glitters Is Gold: Metal-Migration-Induced Degradation in Perovskite Solar Cells. ACS Nano 2016, 10, 6306-6314.

(45) Wei, D.; Wang, T.; Ji, J.; Li, M.; Cui, P.; Li, Y.; Li, G.; Mbengue, J. M.; Song, D. Photo-Induced Degradation of Lead Halide Perovskite Solar Cells Caused by the Hole Transport Layer/Metal Electrode Interface. J. Mater. Chem. A 2016, 4, 1991-1998.

(46) Jeong, B.; Han, H.; Park, C. Micro- and Nanopatterning of Halide Perovskites where Crystal Engineering for Emerging Photoelectronics Meets Integrated Device Array Technology. Adv. Mater. 2020, 32, 2000597.

(47) Deng, Y.; Wang, Q.; Yuan, Y.; Huang, J. Vividly Colorful Hybrid Perovskite Solar Cells by Doctor-Blade Coating with Perovskite Photonic Nanostructures. Mater. Horiz. 2015, 2, 578-583.

(48) Hu, Q.; Zhao, L.; Wu, J.; Gao, K.; Luo, D.; Jiang, Y.; Zhang, Z.; Zhu, C.; Schaible, E.; Hexemer, A.; Wang, C.; Liu, Y.; Zhang, W.; Grätzel, M.; Liu, F.; Russell, T. P.; Zhu, R.; Gong, Q. In situ Dynamic Observations of Perovskite Crystallisation and Microstructure
Evolution Intermediated from $\left[\mathrm{PbI}_{6}\right]^{4-}$ Cage Nanoparticles. Nat. Commun. 2017, 8, 15688.

(49) Bangsund, J. S.; Fielitz, T. R.; Steiner, T. J.; Shi, K.; Van Sambeek, J. R.; Clark, C. P.; Holmes, R. J. Formation of Aligned Periodic Patterns during the Crystallization of Organic Semiconductor Thin Films. Nat. Mater. 2019, 18, 725-731.

(50) Wu, Y.; Islam, A.; Yang, X.; Qin, C.; Liu, J.; Zhang, K.; Peng, W.; Han, L. Retarding the Crystallization of PbI2 for Highly Reproducible Planar-Structured Perovskite Solar Cells via Sequential Deposition. Energy Environ. Sci. 2014, 7, 2934-2938.

(51) Miyamae, H.; Numahata, Y.; Nagata, M. The Crystal Structure Of Lead(II)Iodide-Dimethylsulphoxide(1/2), $\mathrm{PbI}_{2}(\mathrm{DMSO})_{2}$. Chem. Lett. 1980, 9, 663-664.

(52) Cao, X.; Zhi, L.; Jia, Y.; Li, Y.; Zhao, K.; Cui, X.; Ci, L.; Zhuang, D.; Wei, J. A Review of the Role of Solvents in Formation of HighQuality Solution-Processed Perovskite Films. ACS Appl. Mater. Interfaces 2019, 11, 7639-7654.

(53) Bercegol, A.; Ramos, F. J.; Rebai, A.; Guillemot, T.; Puel, J.-B.; Guillemoles, J.-F.; Ory, D.; Rousset, J.; Lombez, L. Spatial Inhomogeneity Analysis of Cesium-Rich Wrinkles in Triple-Cation Perovskite. J. Phys. Chem. C 2018, 122, 23345-23351.

(54) Zheng, G.; Zhu, C.; Ma, J.; Zhang, X.; Tang, G.; Li, R.; Chen, Y.; Li, L.; Hu, J.; Hong, J.; Chen, Q.; Gao, X.; Zhou, H. Manipulation of Facet Orientation in Hybrid Perovskite Polycrystalline Films by Cation Cascade. Nat. Commun. 2018, 9, 2793.

(55) Lin, C. Y.; Li, S. S.; Chang, J. W.; Chia, H. C.; Hsiao, Y. Y.; Su, C. J.; Lian, B. J.; Wen, C. Y.; Huang, S. K.; Wu, W. R.; Wang, D. Y.; Su, A. C.; Chen, C. W.; Jeng, U. S. Unveiling the Nanoparticle-Seeded Catalytic Nucleation Kinetics of Perovskite Solar Cells by TimeResolved GIXS. Adv. Funct. Mater. 2019, 29, 1902582.

(56) Liu, M.; Chen, Y.; Tan, C.-S.; Quintero-Bermudez, R.; Proppe, A. H.; Munir, R.; Tan, H.; Voznyy, O.; Scheffel, B.; Walters, G.; Kam, A. P. T.; Sun, B.; Choi, M.-J.; Hoogland, S.; Amassian, A.; Kelley, S. O.; García de Arquer, F. P.; Sargent, E. H. Lattice Anchoring Stabilizes Solution-Processed Semiconductors. Nature 2019, 570, 96101.

(57) Hernandez Oendra, A. C.; De Leo, E.; Koepfli, S. M.; Winkler, J. M.; Rossinelli, A. A.; Riedinger, A.; Norris, D. J.; Prins, F. Template Stripping of Perovskite Thin Films for Dry Interfacing and Surface Structuring. ACS Appl. Mater. Interfaces 2020, 12, 26601-26606. 